\title{
Beginning of Earthquakes Modeled with the Griffith's Fracture Criterion
}

\author{
by Tamao Sato and Hiroo Kanamori
}

\begin{abstract}
We present a source model for the beginning of earthquakes based on the Griffith's fracture criterion. The initial state is a critical state of pre-existing circular fault, which is on the verge of instability. After the onset of instability, the fault grows with a progressively increasing rupture speed, satisfying the condition of fracture energy balance at the crack tip. We investigate the difference in rupture growth patterns in two classes of models that are considered to represent end-member cases. In the first model (Spontaneous model), we assume that the surface energy varies smoothly as a function of position in the crust. In this model, faults with small initial dimensions grow in regions with small surface energy, and those with large initial dimensions, in large surface energy. The rupture velocity increases progressively until it reaches its limiting value. The synthetic velocity seismogram at far field shows a weak initial phase during the transitional stage to limiting velocity. The time taken to reach the limiting velocity is proportional to the initial length of preexisting fault. Therefore, the duration of the weak initial phase scales with the initial length of fault. In the second model (Trigger model), we envisage that there are many pre-existing faults in the crust with various lengths. These faults are stable because they encounter some obstacle at their ends (e.g., fault segmentation, strong asperity, etc.). This situation is modeled with a local increase of surface energy near the ends of fault. An earthquake is triggered when the obstacle is suddenly removed (i.e., sudden weakening) or the stress is suddenly increased locally to overcome the obstacle. Once an earthquake is triggered, the fault growth is governed by the ambient surface energy. In this model, the rupture speed attains its limiting velocity almost instantly. The synthetic velocity seismogram at far field shows an abrupt, linear increase in amplitude without the weak initial phase that appears in the Spontaneous model. Both models can be unified using a trigger factor defined as a fractional perturbation of the surface energy at the ends of fault relative to the ambient surface energy. The Spontaneous model is characterized by a small trigger factor, and the Trigger model, by a large trigger factor. Thus, the seismic initiation phase with and without the slow initial phase can both occur depending on the trigger factor. The variability in the observed seismic initiation phase may represent a variation of surface energy (strength) distribution surrounding the pre-existing cracks. A theoretical consideration of rupture arrest by barriers using the Griffith's fracture criterion does not support the scaling relation between the nucleation moment and the eventual size of earthquake.
\end{abstract}

\section{Introduction}

Recently, seismologists have paid more attention than ever to the initial part of the seismogram, this time not for merely determining its onset time or polarity but for seeking the possibility of predicting the eventual size of earthquakes from the initial waveform patterns (Iio, 1992, 1995; Umeda, 1990, 1992; Ellsworth and Beroza, 1995). As more studies were done, however, it has become clear that observed seis- mic initiation phases are complex and cannot be represented by a simple scaling relation with the earthquake magnitude (Fukao and Shibazaki, 1995; Ishihara et al., 1992; Mori and Kanamori, 1996; Nakatani et al., 1995; Shibazaki et al., 1994). Furthermore, the discrepancy has become apparent about the interpretation of seismic initiation phases. Earlier, a slow earthquake beginning, termed the slow initial phase 
(Iio, 1992, 1995) or the seismic nucleation phase (Ellsworth and Beroza, 1995), has been interpreted as being due to the source effect. Recently, Mori and Kanamori (1996) attributed the slow initial phases observed in the velocity waveforms recorded during the 1995 Ridgecrest sequence to the attenuation effect. Re-examining the observations of the Ridgecrest sequence, Ellsworth and Beroza (1998) supported the Mori and Kanamori's contension that those earthquakes begin without a slow initial phase of the type observed by Iio (1992, 1995). These reports have raised the question of whether earthquakes are always accompanied by initial slow phases.

In this article, we present a source model for earthquake beginning based on the Griffith's fracture criterion. We investigate the difference in rupture growth patterns of two models that are considered to represent end-member cases. It will be shown that abrupt and slow initial phases can both occur depending on the distribution of strength surrounding the nucleation zone of dynamic rupture.

\section{Earthquake Initiation}

We consider earthquake models governed by the Griffith's fracture criterion (Kostrov, 1966). The essential aspect of this model is that under a given driving stress, $\sigma_{0}$, and surface energy, $\gamma$, of the material, a pre-existing crack is stable until its size reaches a threshold value $l_{0}$. At this point, the crack is in a critical state, and with an infinitesmal increase in $\sigma_{0}$ or decrease in $\gamma$, the crack will grow with a progressively increasing rupture speed, satisfying the energy balance condition at the crack tip. This view of earthquake initiation is consistent with laboratory experiments of stick slip (Dieterich, 1978; Ohnaka and Kuwahara, 1990), in which the zone of quasi-static nucleation process takes the role of pre-existing crack in the present model. The quasistatic process leading to the critical state is beyond the scope of the present study.

The main objective of this study is to provide a simple model to explain the beginning of an earthquake. Of course, because the structure and strength distributions in Earth's crust is complex, an earthquake must be modeled as a multiple crack system either on a single plane or on multiple planes, and its rupture process is expected to be complex because of interactions of many cracks (Umeda, 1992; Umeda et al., 1996; Kame and Yamashita, 1997). The time history of the actual ground motion should be expressed as a superposition of ground motions from individual cracks. Therefore, the single crack model in this article is meant to be used for interpreting the general behavior of the very beginning of an earthquake, during which the effects of structural complexity and multiple crack interactions are considered less important.

Within the framework of this basic concept, we attempt to encompass a wide range of situations by focusing on the heterogenity of fracture strength near the ends of pre-exist- ing cracks. Two classes of models will be considered to represent the end-member cases. In the following, "crack" and "fault" are used interchangeably.

In the first model (Fig. 1), we assume that the surface energy, $\gamma(\mathbf{r})$, varies smoothly as a function of position, $\mathbf{r}$, in the crust that contains pre-existing small faults. The fault near $\mathbf{r}$ has the critical dimension, $l_{0}(\mathbf{r})$, determined by the surface energy $\gamma(\mathbf{r})$. Under this condition, an earthquake occurs when either $\sigma_{0}$ suddenly increases or the material weakens (i.e., $\gamma$ decreases) suddenly, and instability occurs. In this model, faults with small initial dimensions grow in the medium with small surface energy, and those with large initial dimensions, in large surface energy. We investigate the differences in the behavior of initial growth of the cracks with different initial length $l_{0}(\mathbf{r})$.

In the second model (Fig. 1), we assume that the surface energy is constant, $\bar{\gamma}$, throughout the crust except near the ends of faults where it is significantly larger than $\bar{\gamma}$. This model simulates a locked fault. We envisage that there are many pre-existing faults in the crust with various length, $l_{0}(\mathbf{r})$. These faults are stable because they encounter some obstacle at their ends (e.g., fault segmentation, strong asperity, etc.). This situation is modeled with a local increase in the surface energy near the ends of faults. In this model, an earthquake is triggered when the obstacle is suddenly removed (i.e., sudden weakening) or the stress is suddenly increased locally to overcome the obstacle. Once an earthquake is triggered, then the fault growth is governed by the Griffith's fracture criterion in a medium with $\bar{\gamma}$. In contrast to the first model, in this model, both short and long faults grow, after having been triggered, in the medium with the same surface energy, $\bar{y}$.
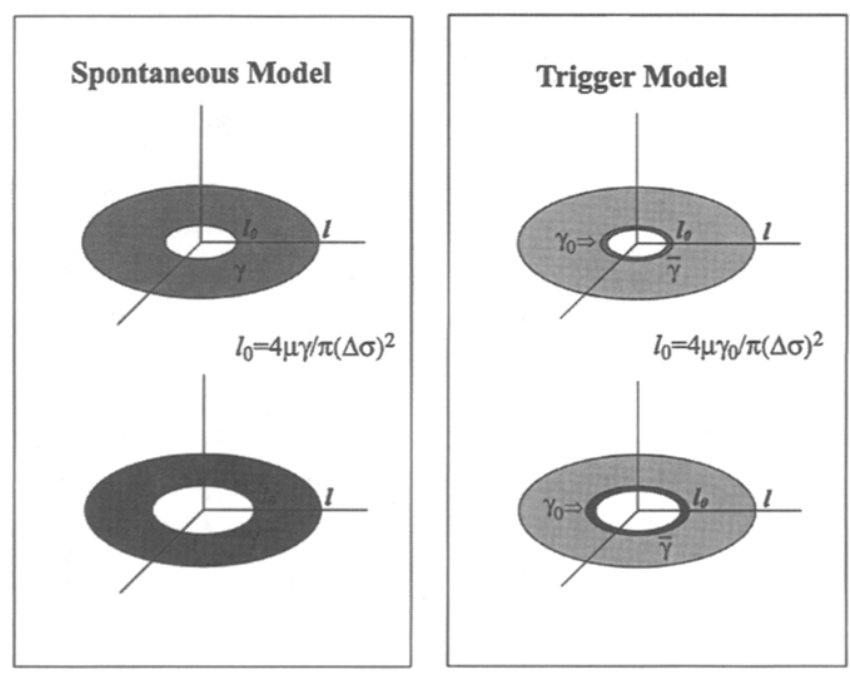

Figure 1. Illustration of two end-member models of earthquake beginning. The trigger model differs from the Spontaneous model by the presence of strong obstacle at the ends of pre-existing crack. 
We investigate the difference in fault growth patterns in these two models. The first model may be called "Spontaneous model," and the second "Trigger model."

\section{Dynamic Rupture of Pre-existing Crack}

Rose (1976) studied the problem of determining the motion of antiplane (mode III) crack under the initial condition that the pre-existing crack is in a critical state before the instability occurs. Following Rose (1976), we formulate the equation of motion of antiplane crack for a generalized case in which the residual frictional stress has a nonzero value. Let $l_{0}$ denote the half-length of pre-existing crack, $\sigma_{0}$ the uniform applied stress, and $\sigma_{d}$ the residual frictional stress on the crack plane. Then the stress field around the preexisting crack is (Knopoff, 1958) given by

$$
\sigma=\sigma_{d}+p(x), \quad|x|>l_{0}
$$

where $p(x)$ is

$$
p(x)=\frac{|x|\left(\sigma_{0}-\sigma_{d}\right)}{\left(x^{2}-l_{0}^{2}\right)^{1 / 2}} .
$$

Suppose that the position of both crack tips is given by $x= \pm l(t)$ with the time $t$ measured from that instant at which the crack begins to extend. In the following analysis, we shall find it convenient to use the normalized variables $\zeta$ and $t^{*}$ defined by

$$
\begin{aligned}
\zeta & =\left(l-l_{0}\right) / l_{0}, \\
t^{*} & =t /\left(l_{0} / \beta\right),
\end{aligned}
$$

where $\beta$ is the shear-wave velocity.

In the Griffith's fracture criterion, the equation of cracktip motion is expressed as

$$
G=2 \gamma
$$

where $\gamma$ is the surface energy and $G$ is the crack extension force, or strain energy release rate, per unit of new crack separation area formed during an increment of crack extension.

The crack extension force on an arbitrarily moving antiplane crack is (Kostrov, 1966) given by

$$
G=\frac{K^{2}}{2 \mu}\left(1-v^{2} / \beta^{2}\right)^{-1 / 2},
$$

where $\mu$ denotes the rigidity, $v$ is the instantaneous crack speed, and $K$ is the dynamic stress intensity factor that depends on the instantaneous crack speed and a static factor $K^{*}$ through

$$
K=(1-v / \beta)^{1 / 2} K^{*} .
$$

At the beginning of the motion,

$$
K^{*}=\sqrt{2 / \pi} \int_{l-\beta t}^{l} \frac{p[x, t-(l-x) / \beta]}{\sqrt{l-x}} d x .
$$

After the disturbance produced either by one of the moving tips reaching the other, a correction term must be added to equation (7) to account for the effect of this disturbance. Although the correction term forces the crack-tip motion to accelerate toward its limiting speed somewhat faster (Rose, 1976), this effect is considered to be minor in the present study. Therefore, we disregard the correction term and assume that $K^{*}$ is given by equation (7) during the entire rupture process.

On evaluating the integral in (7) with $p(x)$ given by (2), we find that

$$
K^{*}=K_{0} U(\zeta)
$$

with

$$
K_{0}=\left(\sigma_{0}-\sigma_{d}\right)\left(\pi l_{0}\right)^{1 / 2}
$$

and

$$
U(\zeta)=(2 / \pi)\{(2+\zeta) E+F\} /(1+\zeta / 2)^{1 / 2} .
$$

$F$ and $E$ in (10) denote the complete elliptic integrals of the first and second kind,

$$
\begin{aligned}
& F(k)=\int_{0}^{\pi / 2} \frac{d \theta}{\sqrt{1-k^{2} \sin ^{2} \theta}}, \\
& E(k)=\int_{0}^{\pi / 2} \sqrt{1-k^{2} \sin ^{2} \theta} d \theta,
\end{aligned}
$$

with modulus $k=\{\zeta /(2+\zeta)\}^{1 / 2}$.

Using the relation between $G$ and $K^{*}$ given by (5), (6), and (8), we can rewrite the equation of motion (4) as

$$
\dot{\zeta}=\left\{U^{4}-\left(K_{c} / K_{0}\right)^{4}\right\} /\left\{U^{4}+\left(K_{c} / K_{0}\right)^{4}\right\},
$$

where $\dot{\zeta}\left(\equiv d \zeta / d t^{*}\right)$ denotes the crack speed normalized by shear-wave velocity $\beta$, and $K_{c}$ is the fracture toughness, $K_{c}=(4 \mu \gamma)^{1 / 2}$. If we allow the surface energy to vary with $\zeta,\left(K_{0} / K_{c}\right)$ in (12) is not constant.

The apparent singularity of the stress field given by (2) is simply a mathematical expression of the stress concentration and is not always inconsistent with the actual finite stress around the crack tip. It should be emphasized that although the shear stress is infinite at the crack tip, the energy flux into the tip is finite. In a simple slip-weakening model, twice the surface energy is identified with the work done in 
the rupture breakdown, or cohesive, zone in excess of that done against the residual frictional stress (Ida, 1972; Palmer and Rice, 1973; Day, 1982). The slip-weakening model is equivalent to the Griffith's fracture criterion in its prediction of rupture growth, provided the zone over which the cohesive tractions act is small compared with overall crack dimension (Ida, 1972). It is demonstrated (Andrews, 1985) that the large-scale numerical solution for traction and slip velocity agreed well with the analytic solution and that the small-scale solution in the smeared-out crack tip adjusted itself to absorb the same energy as absorbed at the singularity in the analytic solution.

In the Spontaneous model, we assume that the surface energy, $\gamma(\mathbf{r})$, varies smoothly as a function of position, $\mathbf{r}$, in the crust; that means, $\gamma$ can vary from event to event, but it is constant during the respective events. The crack is in a critical state when $K_{0}=K_{c}$. Since $U(\zeta \rightarrow 0)=1$, it follows from (12) that the crack speed at $t^{*}=0$ is zero. Therefore, no crack extension occurs in the critical state. However, a perturbation that makes $K_{0}>K_{c}$ will lead to unstable growth of the crack. Let $\delta$ define the perturbation of infinitesmally small magnitude through

$$
\left(K_{0} / K_{c}\right)^{2}=1+\delta .
$$

Because $K_{c}$ in the Spontaneous model is assumed to be constant along the fault, we can rewrite the equation of motion (12) using the relation given by (13):

$$
\dot{\zeta}=\left\{U^{4}(1+\delta)^{2}-1\right\} /\left\{U^{4}(1+\delta)^{2}+1\right\} .
$$

Integrating (14), we obtain

$$
t^{*}=\zeta+2 \int_{0}^{\zeta} \frac{d \zeta}{U^{4}(1+\delta)^{2}-1}
$$

Note that the crack-tip motion is expressed with normalized variables defined by (3).

In the Trigger model, we assume that the surface energy is constant, $\bar{\gamma}$, throughout the crust except near the ends of faults where it is significantly larger than $\bar{\gamma}$. As an extreme case, we assume that the heterogeneity is concentrated at the ends of pre-existing crack $x= \pm l_{0}$. Let $\gamma_{0}$ denote the surface energy at the ends of a pre-existing crack and $\eta$ represent a fractional perturbation of $\gamma_{0}$ relative to the ambient surface energy $\bar{\gamma}$; that is,

$$
\eta=\left(\gamma_{0}-\bar{\gamma}\right) / \bar{\gamma} .
$$

Then the ratio between the fracture toughness at the ends of the pre-existing crack, $K_{c}^{\prime}=\left(4 \mu \gamma_{0}\right)^{1 / 2}$, and the fracture toughness throughout the crust, $K_{c}=(4 \mu \bar{\gamma})^{1 / 2}$, is given by

$$
\left(K_{c}^{\prime} / K_{c}\right)^{2}=1+\eta
$$

The initial crack is in a critical state when $K_{0}=K_{c}^{\prime}$. The slightest perturbation that makes $K_{0}>K_{c}^{\prime}$ will cause instability. Let

$$
\left(K_{0} / K_{c}^{\prime}\right)^{2}=1+\delta^{\prime}
$$

where $\delta^{\prime}$ is an infinitesmally small positive number. Using (17) and (18), we find

$$
\left(K_{0} / K_{c}\right)^{2}=1+\delta
$$

with

$$
\delta=\delta^{\prime}+\left(1+\delta^{\prime}\right) \eta
$$

For $\eta \gg \delta^{\prime},(20)$ is approximated by

$$
\delta \simeq \eta
$$

Note that the equation of motion (14) in the Trigger model is represented with $\delta$ given by (20), which is no longer infinitesmally small. It can take a large value depending on $\eta$. The Spontaneous model is considered a special case where $\eta=0$. In terms of $\delta$, which we call the "trigger factor" hereafter, the Spontaneous model is associated with an infinitesmally small trigger factor and the Trigger model with a large trigger factor. The difference in the magnitude of trigger factor yields a different mode of rupture growth, as shown in the following.

In the Trigger model, we assumed that the surface energy is constant throughout the crust except at the ends of pre-existing cracks. This assumption is not essential because as long as $\gamma_{0}>\bar{\gamma}$, the rupture can propagate outward. Thus, we regard $\bar{\gamma}$ as the surface energy of the respective events.

Figure 2 shows the time taken by a crack tip to advance

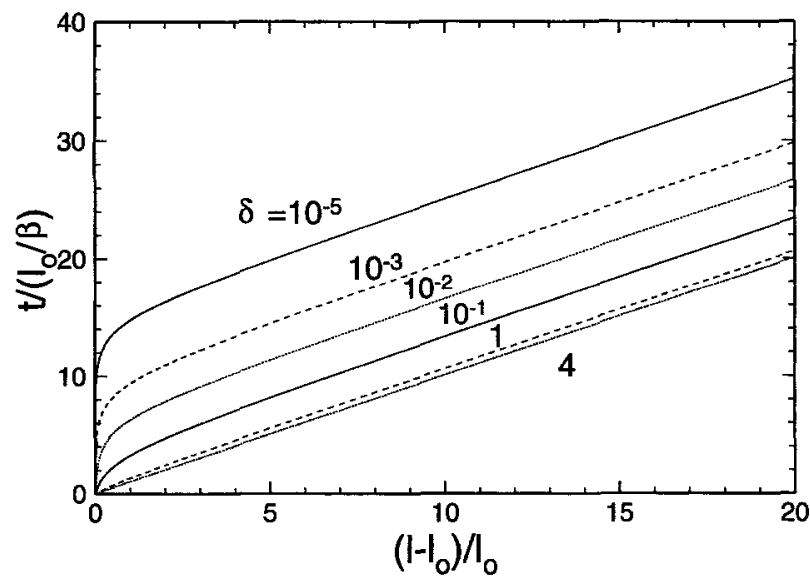

Figure 2. Plots of time versus crack-tip position for different trigger factors, $\delta$. They are shown in normalized units of time and position. $\beta$ is the shear-wave velocity, and $l_{0}$ is the half-length of pre-existing crack. 
by a fraction $\zeta$ of the original crack half-length. It is calculated using (15) for various $\delta$. The time taken by a crack tip to reach a given position $\zeta$ increases with decreasing $\delta$. For greater values of $\delta$, the position of crack tip increases linearly with time from the very beginning. The crack speed for the linear slope is almost equal to the shear-wave velocity, which is the limiting crack speed for the two-dimensional antiplane crack. The changes of crack speed versus time are demonstrated in Figure 3. The time for the crack to attain the limiting crack speed gets longer with decreasing $\delta$. Since the Spontaneous model is defined as having a small $\delta$ and the Trigger model having a large $\delta$, it follows that the Spontaneous model is characterized by a slow increase and the Trigger model by an abrupt increase in crack speed toward the limiting value. Figure 4 exhibits the crack speed as a function of $\zeta$. It is shown that the crack speed for a given value of $\zeta$ is practically unaffected by the value of $\delta$ for $\delta<10^{-2}$.

We found earlier that the equation of crack-tip motion is expressed with the normalized variables $t^{*}$ and $\zeta$. Its implication becomes clear when we show the crack-tip motion in real units of time and length (Fig. 5). For a small $\delta$, say, less than $10^{-1}$, the time taken by a crack tip to advance by a given distance, $l-l_{0}$, increases with increasing half-length of pre-existing crack, $l_{0}$. In other words, the pre-existing cracks with larger $l_{0}$ grows less rapidly than those with smaller $l_{0}$. This scaling relation is characteristic of the Spontaneous model. Using (4) through (9), we obtain

$$
l_{0}=\frac{4 \mu \gamma}{\pi\left(\sigma_{0}-\sigma_{d}\right)^{2}}
$$

Therefore, pre-existing cracks with small lengths grow in the medium with small surface energy, and those with large lengths, in larger surface energy, provided that the stress drop $\left(\sigma_{0}-\sigma_{d}\right)$ is constant.

In the Trigger model, the surface energy at the ends of pre-existing crack is given by (16). Putting this into (22), we have

$$
l_{0}=(1+\eta) \bar{l}_{0},
$$

where $\bar{l}_{0}$ denotes the critical half-length of pre-existing crack for the constant surface energy, $\bar{\gamma}$. Pre-existing cracks locked by larger surface energy at their ends grow from larger initial sizes. From (21) and (23), we get

$$
\delta \simeq l_{0} / \bar{l}_{0}-1
$$

Thus, we find that $\delta$ is proportional to the ratio of $l_{0}$ to $\bar{l}_{0}$. As an example, we show in Figure 6 the crack-tip motion for $l_{0}$ ranging 5 to $25 \mathrm{~m}$ with $\bar{l}_{0}=1 \mathrm{~m}$. For these values, $\delta$ given by (24) ranges from 4 to 24 . The crack immediately grows with the limiting crack speed for all cases.

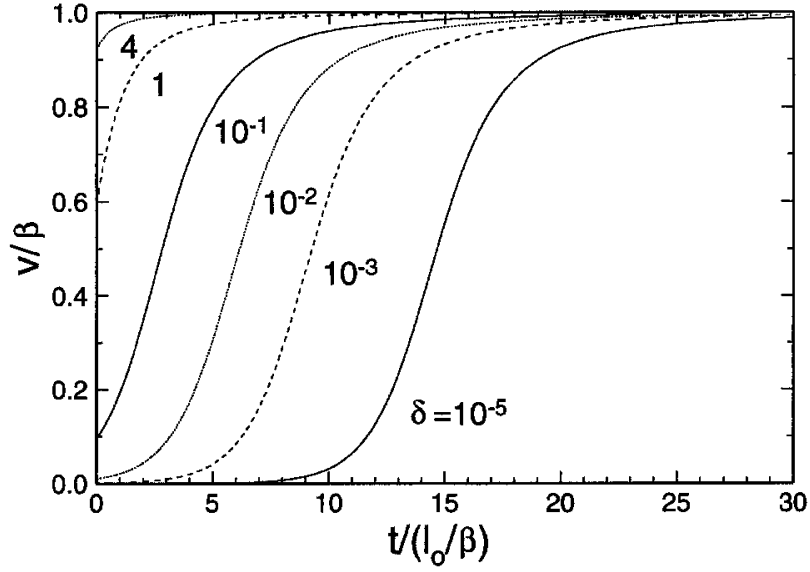

Figure 3. Plots of crack speed versus time for different trigger factors, $\delta$. They are shown in normalized units of crack speed and time.

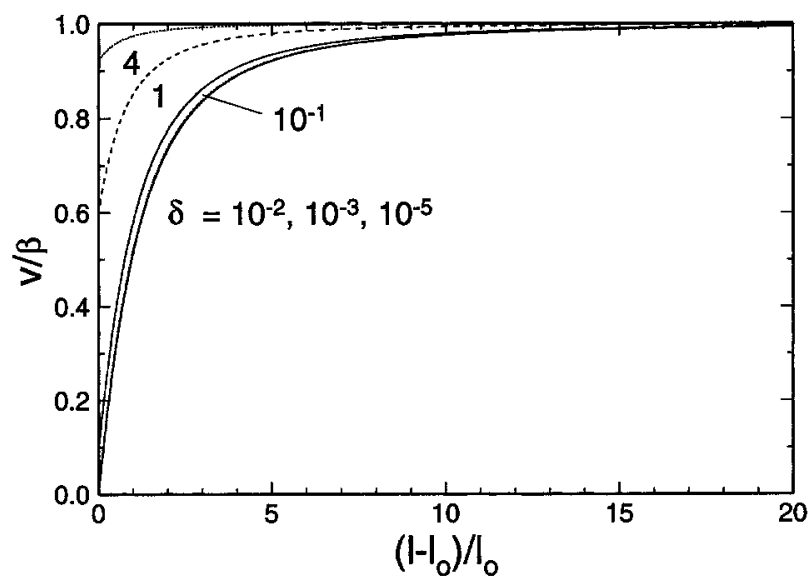

Figure 4. Plots of crack speed versus crack-tip position for different trigger factors, $\delta$. They are demonstrated in normalized units.

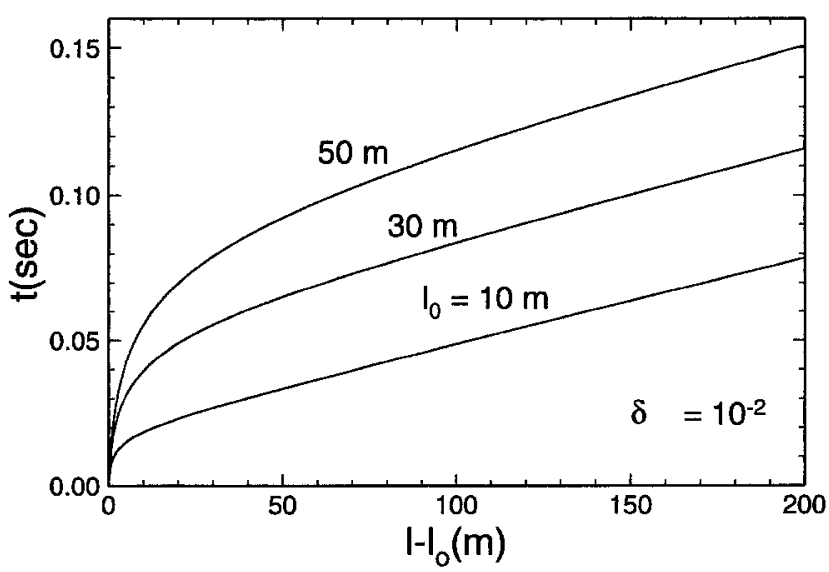

Figure 5. Plots of time versus crack-tip position in real units of time and length for the Spontaneous model $\left(\delta=10^{-2}\right)$. 


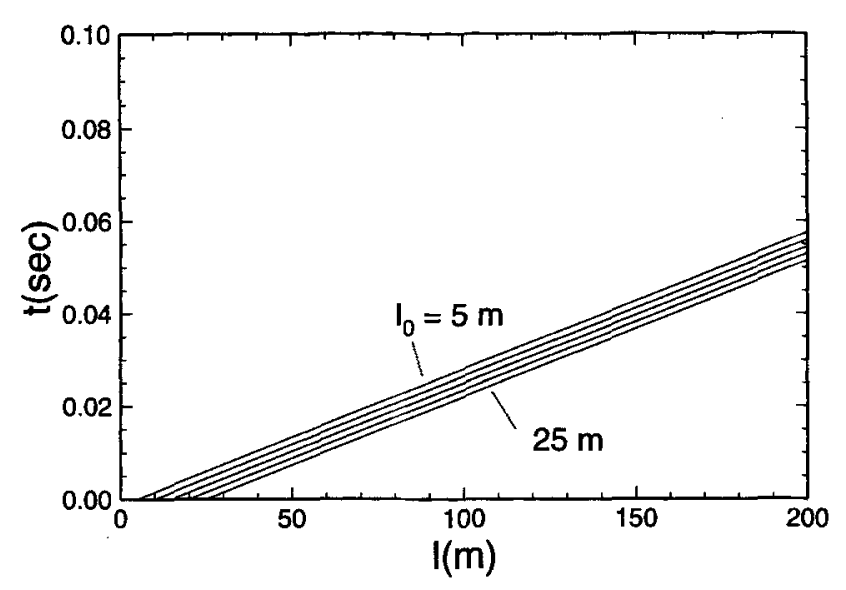

Figure 6. Plots of time versus crack-tip position in real units of time and length for the Trigger model. $l_{0}$ varies from 5 to 25 by a step of $5 \mathrm{~m}$. The trigger factor is given by equation (24) with $\bar{l}_{0}=1 \mathrm{~m}$.

\section{Seismic Initiation Phase}

If our primary interest is on the details of seismic radiation due to fault motion, we must deal with actual faults that have finite dimensions three dimensionally. Previously, Virieux and Madariaga (1982) solved a similar problem of dynamic rupture of a pre-existing circular fault using a finitedifference method. But their study was not centered on investigating the properties of seismic initiation phases radiated from the dynamic rupture. In this study, we do not employ such a numerical approach as taken by Virieux and Madariaga (1982). Instead, we assume a kinematic source model in which a pre-existing crack of circular geometry expands circularly with the rupture velocity obtained for the two-dimensional antiplane crack in the previous section. Several numerical simulations show that the three-dimensional spontaneous rupture involves a directional variation of rupture velocity (Miyatake, 1980; Das, 1981; Day, 1982; Virieux and Madariaga, 1982; Andrews, 1994). However, the results of those simulations also indicate that the shape of rupture front does not heavily depart from a circular geometry during the earlier stage of rupture. Given the time history of the crack-tip motion, we compute the seismic initiation phase using Sato's (1994) method, which is based on the assumption that the slip on the crack should comply at all times with Eshelby's (1957) static solution. It is (Sato, 1994) shown that this assumption on the slip gives a good approximation to the self-similar slip distribution obtained by Kostrov (1964) for the case of constant rupture velocity. In this way, we can calculate radiated seismic waves efficiently, which is otherwise tedious, while preserving the basic properties of three-dimensional dynamic rupture.

We assume an infinite, isotropic, homogeneous medium with a Poisson's ratio of $1 / 4$. If we let the $\left(x_{1}, x_{2}\right)$ plane be the fault plane, and the positive $x_{1}$ axis be the direction of the relative slip, the far-field displacements of $P$ and $S$ waves are expressed by (Haskell, 1964)

$$
\begin{aligned}
& u_{r}=\frac{(\beta / \alpha)^{3} \sin 2 \theta \cos \phi}{4 \pi \mu \beta r_{0}} \dot{M}_{c}(t), \\
& u_{\theta}=\frac{\cos 2 \theta \cos \phi}{4 \pi \mu \beta r_{0}} \dot{M}_{c}(t) \\
& u_{\phi}=\frac{-\cos \theta \sin \phi}{4 \pi \mu \beta r_{0}} \dot{M}_{c}(t)
\end{aligned}
$$

where $\dot{M}_{c}(t)$ is the apparent moment rate function, $c$ stands for $\alpha$ or $\beta$ (the compressional and shear-wave velocities) according to the particular wave under consideration, and $r_{0}$ denotes the distance from the center of the fault to the observer. The displacements $u_{r}, u_{\theta}$, and $u_{\phi}$ denote $P, S V$, and $S H$ waves, respectively, where the directions of shear-wave motion are defined relative to the fault plane, $\theta$ is the takeoff angle measured from the fault normal, and $\phi$ is the azimuth. For a Poisson solid, $\alpha^{2} / \beta^{2}=3$.

On the assumption that the slip should comply at all times with Eshelby's (1957) static solution for the constant shear stress drop on the fault, the apparent moment rate function for a circular crack expanding with variable rupture velocity is given by (Sato, 1994)

$$
\dot{M}_{c}(\tau)=\frac{24 \Delta \sigma}{7} \frac{L_{a}(\tau)^{2}-L_{b}(\tau)^{2}}{2 q}
$$

where

$$
\begin{aligned}
\tau & =t-r_{0} / c, \\
q & =\frac{\sin \theta}{c}
\end{aligned}
$$

Because the assumption on the slip is considered a good approximation to the solution of dynamic rupture, we may regard the stress drop, $\Delta \sigma$, in (26) as being equal to the dynamic stress drop that corresponds to $\sigma_{0}-\sigma_{d}$ in (2). $L_{a}(\tau)$ and $L_{b}(\tau)$ are the lengths of the nearest and the most distant isochrones from the observer. Let $T(l)$ denote the time when the radius of crack tip reaches $l$, then the seismic waves radiated from the nearest and farthest crack tips arrive at

$$
\begin{aligned}
& T_{a}(l)=T(l)-q l, \\
& T_{b}(l)=T(l)+q l,
\end{aligned}
$$

respectively. Solving $T_{a}(l)=\tau$ and $T_{b}(l)=\tau$ for $l$, we obtain $L_{a}(\tau)$ and $L_{b}(\tau)$ for a given $T(l)$. Here we assume that the crack-tip motion of the circular crack is given by the solution (15) for the two-dimensional antiplane crack in the previous section. Because the crack-tip motion is expressed with the normalized variables, we shall find it convenient to rewrite (28) as 


$$
\begin{aligned}
& \frac{T_{a}(l)}{l_{0} / \beta}=\frac{T(l)}{l_{0} / \beta}-\beta q \frac{l}{l_{0}}, \\
& \frac{T_{b}(l)}{l_{0} / \beta}=\frac{T(l)}{l_{0} / \beta}+\beta q \frac{l}{l_{0}} .
\end{aligned}
$$

Substituting $t^{*}(\zeta)$ of (15) into $T(l) /\left(l_{0} / \beta\right)$ in (29), we solve for $l / l_{0}$ as a function of normalized time $\tau^{*}\left(=\beta \tau / l_{0}\right)$. The solutions are normalized isochrone lengths defined by

$$
\begin{aligned}
& L_{a}^{*}\left(\tau^{*}\right)=L_{a}\left(\tau^{*}\right) / l_{0}, \\
& L_{b}^{*}\left(\tau^{*}\right)=L_{b}\left(\tau^{*}\right) / l_{0} .
\end{aligned}
$$

The apparent moment rate function is then given by

$$
\dot{M}_{c}\left(\tau^{*}\right)=\frac{24 \Delta \sigma l_{0}^{2} \beta}{7} D_{c}\left(\tau^{*}\right)
$$

with

$$
D_{c}\left(\tau^{*}\right)=\frac{L_{a}^{*}\left(\tau^{*}\right)^{2}-L_{b}^{*}\left(\tau^{*}\right)^{2}}{2 \beta q}
$$

Whereas the ground displacement is proportional to $\dot{M}_{c}\left(\tau^{*}\right)$, the ground velocity is proportional to $\ddot{M}_{c}\left(\tau^{*}\right)$, which is given by

$$
\ddot{M}_{c}\left(\tau^{*}\right)=\frac{24 \Delta \sigma l_{0} \beta^{2}}{7} \dot{D}_{c}\left(\tau^{*}\right)
$$

Note that $\dot{D}_{c}\left(\tau^{*}\right)$ indicates the derivative of $D_{c}\left(\tau^{*}\right)$ with respect to $\tau^{*} . D_{c}\left(\tau^{*}\right)$ and $\dot{D}_{c}\left(\tau^{*}\right)$ are both nondimensional quantities and depend only on $q$ and $\delta$.

Figure 7 shows the ground velocities of $P$ waves for different values of $\delta$ at $\theta=30^{\circ}$. Waveforms for other takeoff angles are similar except for a change due to the directivity effect. The rupture is forced to stop at $\zeta=20$ abruptly. The origin of time axis coincides with the arrival time of the initial $P$ wave. For smaller values of $\delta$, the amplitude rises slowly at the onset. The weak initial phase is followed by a linearly growing phase, the slope of which is comparable to that expected for the crack expanding at a constant speed of the shear-wave velocity. As $\delta$ approaches unity, the amplitude increases linearly from the start without a weak initial phase. These results indicate that the Spontaneous model is characterized by a weak, slow phase at the onset, whereas the Trigger model is characterized by a fast rise of amplitude from the beginning.

We showed earlier that the time taken by a crack tip to advance by a fraction $\zeta$ increases as the value of $\delta$ decreases (Fig. 2). This effect of $\delta$ on the initial rupture growth explains why the duration of weak initial phase gets longer as $\delta$ gets smaller. It is noted that, when the waveforms with an appreciable amplitude are compared, the seismic initiation phases calculated for $\delta$ less than, say, $10^{-2}$, are similar to

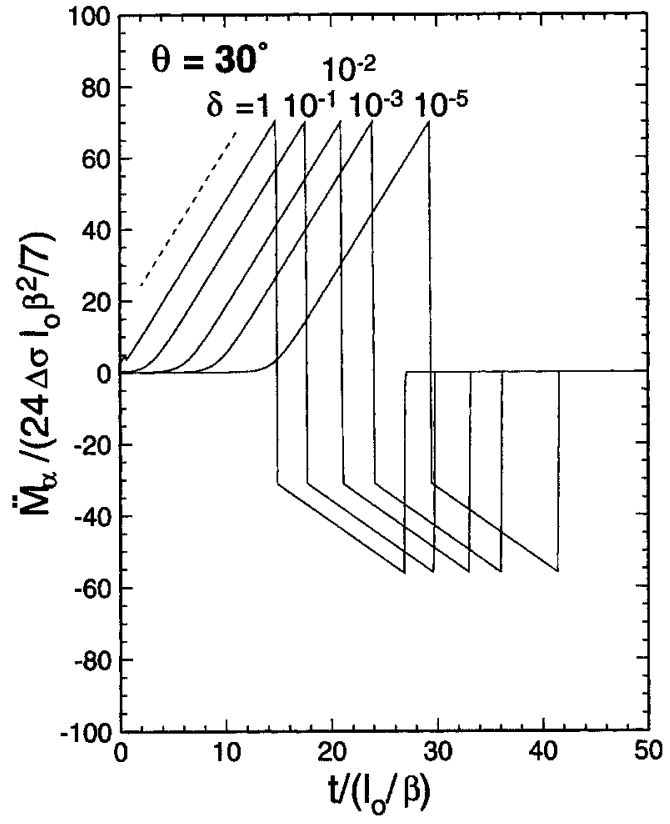

Figure 7. Velocity seismograms at $\theta=30^{\circ}$ for different $\delta$. The ordinate corresponds to $\dot{D}_{c}\left(\tau^{*}\right)$ in equation (33). It is assumed that the crack tip stops abruptly at $\zeta=20$. The dotted line indicates the slope expected for a circular crack growing at a speed of the shear-wave velocity.

each other. This corresponds to the fact that the crack speed for a given value of $\zeta$ is practically independent of $\delta<10^{-2}$ (Fig. 4). Therefore, it is not necessary to consider too small a value of $\delta$, because the weak initial phase of an extremely small amplitude would be masked by the ground noise under the ordinary observational conditions.

From (25), (31), and (33), the $P$-wave displacement and velocity at far field are expressed as

$$
\begin{aligned}
& u_{r}=\frac{6(\beta / \alpha)^{3} \sin 2 \theta \cos \phi}{7 \pi} \frac{\Delta \sigma l_{0}^{2}}{\mu r_{0}} D_{\alpha}\left(\tau^{*}\right) \\
& \dot{u}_{r}=\frac{6(\beta / \alpha)^{3} \sin 2 \theta \cos \phi}{7 \pi} \frac{\Delta \sigma l_{0} \beta}{\mu r_{0}} \dot{D}_{a}\left(\tau^{*}\right)
\end{aligned}
$$

If we put $\alpha / \beta=1.73$ and $\langle\sin 2 \theta \cos \phi\rangle=0.64$, we obtain

$$
\begin{aligned}
& \left\langle u_{r}\right\rangle=0.034 \frac{\Delta \sigma l_{0}^{2}}{\mu r_{0}} D_{a}\left(\tau^{*}\right), \\
& \left\langle\dot{u}_{r}\right\rangle=0.034 \frac{\Delta \sigma l_{0} \beta}{\mu r_{0}} \dot{D}_{a}\left(\tau^{*}\right),
\end{aligned}
$$

where the bracket \langle\rangle denotes the expected value evaluated over the focal sphere.

Figure 8 shows the onsets of velocity seismograms for some specific values of $l_{0}$ and $\Delta \sigma$. We assumed that $\beta=3.4$ $\mathrm{km} / \mathrm{sec}$ and $\mu=3.12 \times 10^{4} \mathrm{MPa}$. The receiver is located at $r_{0}=10 \mathrm{~km}$ and $\theta=52^{\circ}$. We chose $\theta=52^{\circ}$ because $\sin$ 


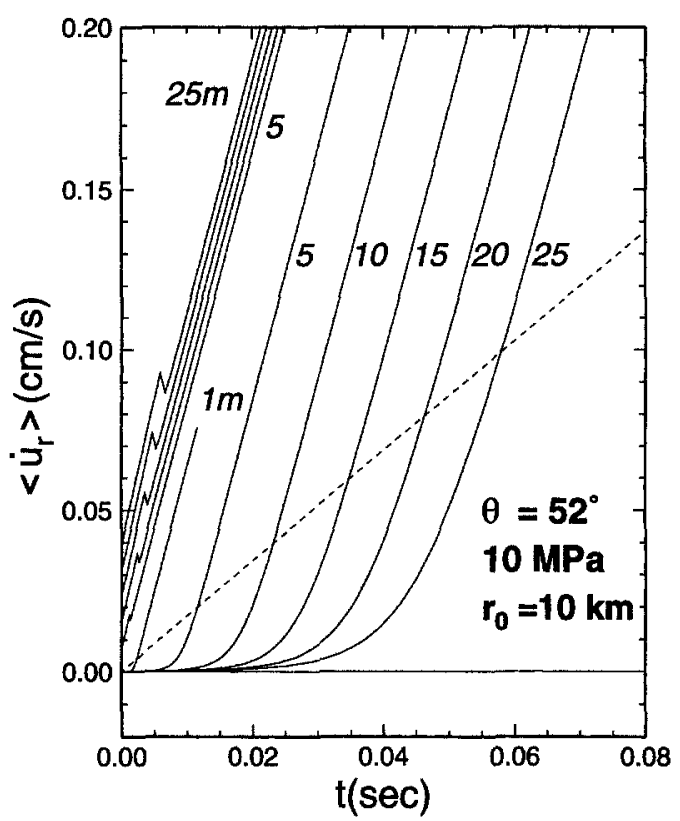

Figure 8. Onsets of velocity seismograms for the Spontaneous model (the group in the right with $l_{0}$ from 1 to $25 \mathrm{~m}$ ) and Trigger model (the group in the left with $l_{0}$ from 5 to $25 \mathrm{~m}$ ). The stress drop $\Delta \sigma=$ $10 \mathrm{MPa}$ is assumed. The observer is located at a takeoff angle $\theta$ of $52^{\circ}$ and a hypocentral distance $r_{0}$ of 10 $\mathrm{km}$. The dotted line indicates the end of slow initial phase defined in the text.

$52^{\circ}$ is equal to the expected value of $\sin \theta$ over the focal sphere. We put $\Delta \sigma=10 \mathrm{MPa}$. The group of seismograms to the right with $l_{0}$ ranging from 1 and $25 \mathrm{~m}$ are calculated for $\delta=10^{-2}$. This group corresponds too the Spontaneous model. The other group of seismograms to the left are synthesized using the parameters for the Trigger model in Figure 6. Among the group of the Spontaneous model, the duration of weak initial phase gets longer as $l_{0}$ increases. The dashed line denotes the locus marking the end of weak initial phase, the definition of which will be explained in the next section. On the other hand, the onsets of seismograms for the Trigger model grow abruptly without weak initial phases for all cases. Instead, we observe a discontinuous change in amplitude, or bump, in the Trigger model. It appears at the time when the wave emitted from the farthest corner of pre-existing crack arrives. The arrival time of the bump is given by

$$
\tau_{b}=\frac{2 l_{0} \sin \theta}{\alpha}
$$

The amplitude of the bump gets larger with increasing $l_{0}$. This is because $\delta$ is proportional to $l_{0}$ in the Trigger model we adopted: The bump becomes more prominent as $\delta$ increases.

Figure 9 shows the far-field displacement for the same parameters as used in Figure 8. In the Spontaneous model,

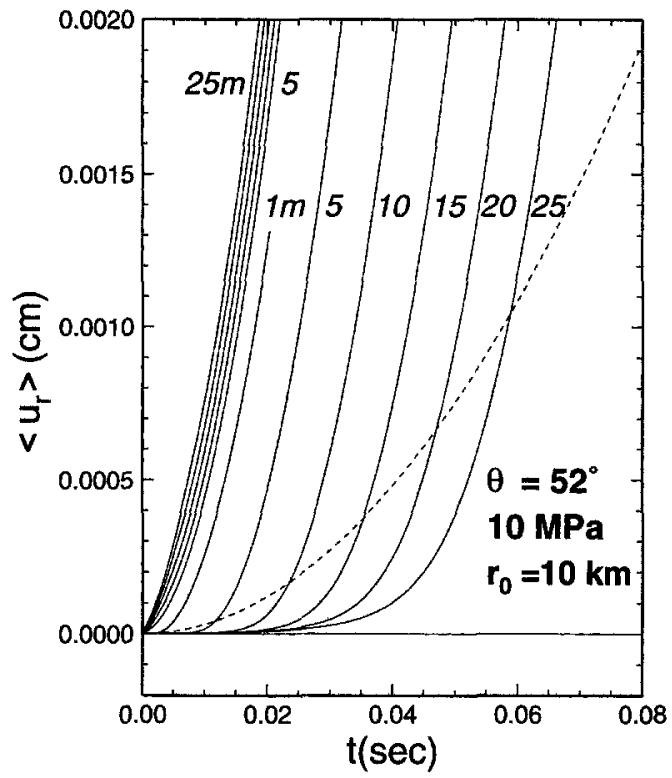

Figure 9. Onsets of displacement seismograms for the Spontaneous model (the group in the right with $l_{0}$ from 1 to $25 \mathrm{~m}$ ) and Trigger model (the group in the left with $l_{0}$ from 5 to $25 \mathrm{~m}$ ). The source parameters and the location of observer are the same as those in Figure 8 . The dotted line indicates the end of slow initial phase defined in the text.

the locus of the end of seismic initiation phase changes quadratically with respect to time. This results from the fact that the displacement is proportional to the square of $l_{0}$, whereas the duration of weak initial phase scales with $l_{0}$. The bumps that appeared in the velocity seismograms for the Trigger model are less obvious in the displacement seismogram.

Iio (1992) was the first to take interest in the emergent onsets in velocity seismograms of microearthquakes and termed the emergent onset the slow initial phase. He suggested that the slow initial phase may be caused by slow slip or slow rupture during the beginning of earthquake (lio, 1992, 1995). Umeda $(1990,1992)$ and Ellsworth and Beroza (1995) also found that earthquakes in a wider range of magnitude begin with a weak initial phase with a low moment relative to the rest of the mainshock. They attributed the socalled seismic nucleation phase to the source effect. On the other hand, Mori and Kanamori (1996) examined the seismic records during the 1995 Ridgecrest sequence and showed that the attenuation effect can explain the curvature in the beginning of velocity waveform without introducing the source effect. Re-examining earthquakes during the 1995 Ridgecrest sequence, Ellsworth and Beroza (1998) supported Mori and Kanamori's conclusion that these earthquakes begin without a resolvable slow initial growth phase of the type reported by lio $(1992,1995)$.

In terms of the present model, the beginning of earthquake with and without the slow initial phase can both occur depending on the trigger factor, $\delta$. The Spontaneous model, or a small $\delta$, is capable of simulating the slow initial phase 
of the type reported by lio $(1992,1995)$, whereas the Trigger model, or a large $\delta$, can explain the abrupt onset of the type reported by Mori and Kanamori (1996). In the present model, the characteristics of seismic initiation phase is governed by the size of pre-existing crack and the trigger factor. If these parameters can vary in different tectonic situations, it is not surprising that we observe seismic initiation phases of various types.

\section{Scaling Law of Slow Initial Phase}

In the previous section, we stated without details that the duration of weak initial phase scales with the size of preexisting crack. Here we define the weak initial phase explicitly and establish the scaling relation in a quantitative manner. Figure 10 shows the initial parts of velocity seismograms for different $\theta$ with $\delta=10^{-2}$. The dotted line indicates the ends of weak initial phases, $\tau_{e}^{*}$, which are defined by the arrival times of waves emitted from the center of a circular crack at the time when the crack tip propagates at a speed of $v / \beta=0.7$. This definition is somewhat arbitrary, but $\tau_{e}^{*}$ thus defined demarcates the boundary between the weak initial phase and a succeeding phase that grows almost linearly. We call the weak initial phase before $\tau_{e}^{*}$ the "slow initial phase" and the succeeding phase simply the "linear phase." Because the crack-tip position for $v / \beta=0.7$ is $\zeta=1.75$ (Fig. 4), the time $t^{*}$ corresponding to $v / \beta=0.7$ is 7.5 (Fig. 2). Therefore, the duration of slow initial phase is given by

$$
\tau_{e}^{*}=7.50+\frac{\sin \theta}{1.73}
$$

where the second term of the right-hand side accounts for the arrival-time difference between the phases radiated at the time of rupture initiation from the center of crack and from the crack tip nearest the receiver. Following the same procedure, we can derive a similar relation for $\delta=10^{-5}$; that is,

$$
\tau_{e}^{*}=16.02+\frac{\sin \theta}{1.73} .
$$

In real unit of time, the duration of slow initial phase, $\tau_{e}$, is represented by

$$
\tau_{e}=\left(l_{0} / \beta\right) \tau_{e}^{*}
$$

Thus, we find that the duration of slow initial phase, or nucleation time, is determined by only $l_{0}$. The nucleation time gets longer as the size of pre-existing crack gets larger. This scaling relation of slow initial phase applies only to the Spontaneous model because the slow initial phase does not appear in the Trigger model.

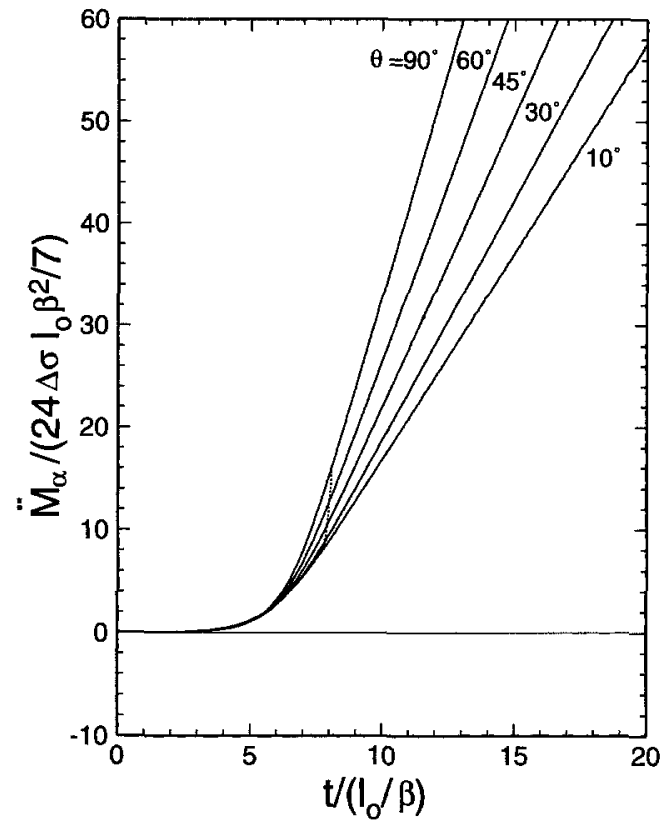

Figure 10. Onsets of velocity seismograms at different $\theta$ for $\delta=10^{-2}$. The dotted line demarcates the boundary between the slow initial phase and the linear phase.

Following Ellsworth and Beroza (1995), we define the nucleation moment as

$$
M_{0}^{v}=\int_{0}^{\tau_{\mathrm{c}}} \dot{M}_{a}(\tau) d \tau
$$

Putting (31) into (40), we obtain

$$
M_{0}^{v}=\frac{24 \Delta \sigma l_{0}^{3}}{7} \int_{0}^{\tau_{e}^{*}} D_{a}\left(\tau^{*}\right) d \tau^{*} .
$$

For $\theta=52^{\circ}$, using (37) and (38), we have $\tau_{e}^{*}=7.96$ for $\delta$ $=10^{-2}$ and $\tau_{e}^{*}=16.48$ for $\delta=10^{-5}$, respectively. On evaluating the integral in (41) for these $\tau_{e}^{*}$, we obtain

$$
\begin{aligned}
& M_{0}^{v}=60.5 \Delta \sigma l_{0}^{3}, \delta=10^{-2} ; \\
& M_{0}^{v}=62.4 \Delta \sigma l_{0}^{3}, \delta=10^{-5} .
\end{aligned}
$$

The nucleation moment is proportional to the dynamic stress drop times the cube of the radius of pre-existing circular crack. The constants of proportionality are almost the same for the two cases. Because the duration of slow initial phases does not strongly depend on take-off angle (Fig. 10), the nucleation moment for other $\theta$ can be approximated by (42). Using the relation given by (39), we can rewrite (42) as

$$
\begin{aligned}
& M_{0}^{v}=4.72 \times 10^{15} \times \Delta \sigma \cdot \tau_{e}^{3}, \delta=10^{-2}, \\
& M_{0}^{v}=0.55 \times 10^{15} \times \Delta \sigma \cdot \tau_{e}^{3}, \delta=10^{-5},
\end{aligned}
$$

where $M_{0}^{\prime}$ is measured in N-m, $\Delta \sigma$ in MPa, and $\tau_{e}$ in sec. 
For the circular crack growing from a point and propagating with a constant rupture velocity, $v$ (Sato and Hirasawa, 1973), the integral of moment rate from the start to a given time, $\tau$, is given by

$$
M_{0}(\tau)=\frac{16 \Delta \sigma v^{3}}{7\left\{1-(v \sin \theta / \alpha)^{2}\right\}^{2}} \tau^{3} .
$$

Putting $v=\beta$ and $\theta=52^{\circ}$ into (44), we have

$$
M_{0}(\tau)=132 \times 10^{15} \times \Delta \sigma \cdot \tau^{3} .
$$

The equation is similar to (43) except that the coefficient of proportionality is much greater than those in (43). Hence, we find that the Spontaneous model is characterized by a slow initial phase that gives an extremely low moment rate relative to the circular crack expanding with a constant crack speed close to the shear-wave velocity.

From the observational point of view, the slow initial phase and nucleation moment should be defined in reference to the level of ground noise. In this attempt, we will neglect the effect of anelastic attenuation because its incorporation hinders an easy, straightforward redefinition of those parameters. Assuming that we lose the slow initial phase with amplitudes less than a given ground noise level, we can determine the start time of slow initial phase, $\tau_{s}^{*}$, using (35). The time that gives the amplitude equal to the given level of ground noise corresponds to $\tau_{s}^{*}$. Then the duration of slow initial phase corrected for the ground noise is given by

$$
\tau_{\vec{d}}^{*}=\tau_{e}^{*}-\tau_{s}^{*} .
$$

If we deal with the velocity seismogram, $\tau_{d}^{*}$ is expressed as a function of $\mu r_{0} \dot{u}_{G} / \Delta \sigma l_{0} \beta$, where $\dot{u}_{G}$ denotes the level of ground noise in velocity. The function is almost independent of $\theta$ for $\mu r_{0} \dot{u}_{G} / \Delta \sigma l_{0} \beta$ less than 0.2 . Figure 11 shows the case for $\theta=52^{\circ}$ and $\delta=10^{-5}$. From the curve in Figure 11, we can derive the relation between the nucleation time and the critial size of pre-existing crack for a particular set of parameters. Figure 12 shows the results for $\dot{u}_{G}=10^{-4} \mathrm{~cm} /$ $\sec , r_{0}=10 \mathrm{~km}$, and $\Delta \sigma=1,10$, and $100 \mathrm{MPa}$. For a given nucleation time, the estimates of $l_{0}$ gets larger with decreasing stress drop.

In conformity with the above modification, the nucleation moment may be redefined as

$$
M_{0}^{v}=\int_{\tau_{s}}^{\tau_{\mathrm{e}}} \dot{M}_{a}(\tau) d \tau
$$

Following the same steps taken in deriving (43), we obtain

$$
M_{0}^{v}=\varepsilon \times \Delta \sigma \cdot \tau_{d}^{3},
$$

where $\varepsilon$ denotes a constant that is given as a function of $\mu r_{0} \dot{u}_{G} / \Delta \sigma l_{0} \beta$. Figure 13 shows the change of $\varepsilon$ for $\theta=52^{\circ}$

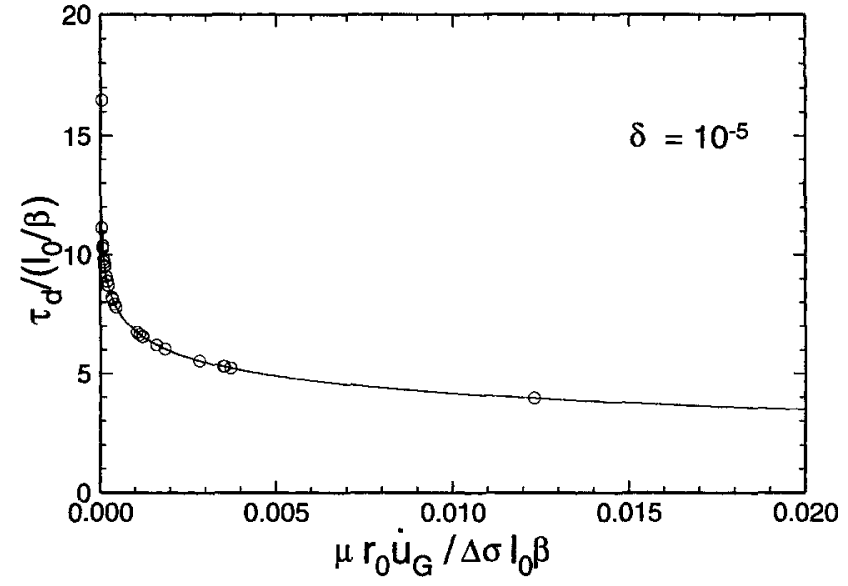

Figure 11. The duration of slow initial phase corrected for the level of ground noise. The theoretical curve is shown with the plots of data taken from $\mathrm{Be}$ roza and Ellsworth (1996).

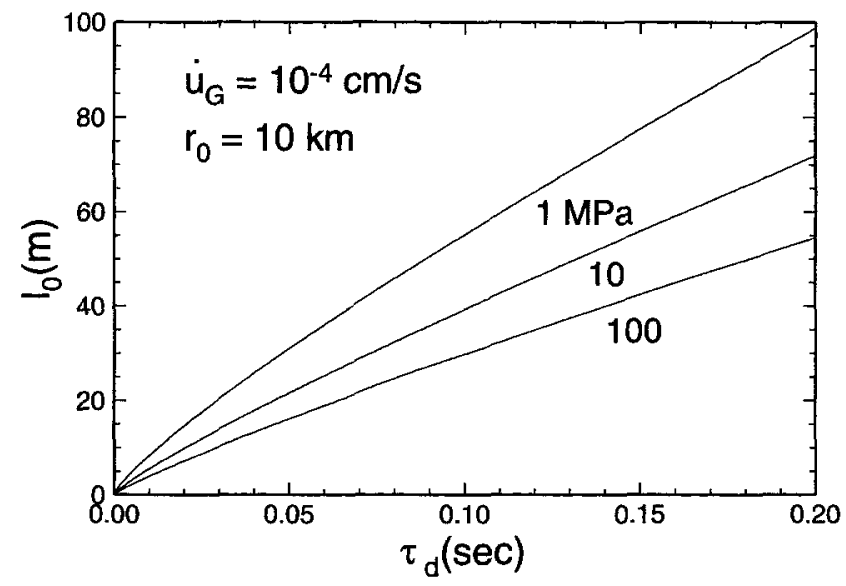

Figure 12. The duration of slow initial phase versus the critical size of pre-existing crack for groundnoise level $\dot{u}_{G}=10^{-4} \mathrm{~cm} / \mathrm{sec}$, hypocentral distance $r_{0}=10 \mathrm{~km}$, and stress drop $\Delta \sigma=1,10$, and 100 $\mathrm{MPa}$.

and $\delta=10^{-5}$. If the ground is perfectly noiseless, that is, $\dot{u}_{G}=0, \varepsilon$ becomes equal to the constant given in (43). On the other hand, if the ground noise is extremely large, thereby making $\mu r_{0} \dot{u}_{G} / \Delta \sigma l_{0} \beta$ large, $\varepsilon$ approaches the constant given in (45).

An important aspect of the Spontaneous model is that as $l_{0}$ increases, it takes longer for the crack expansion speed to reach its limiting speed; consequently, it takes longer for the ground-motion velocity to attain the final linear (in time) trend, as shown by Figure 8. Thus, in the context of this model, we can estimate $l_{0}$ from the duration of the seismic nucleation phase if the dynamic stress drop is known. We apply this concept to the data set obtained by Beroza and Ellsworth (1996). From the theoretical curve of $\tau_{d}^{*}$ versus $\mu r_{0} \dot{u}_{G} / \Delta \sigma l_{0} \beta$ shown in Figure 11, we determined $l_{0}$ from the 


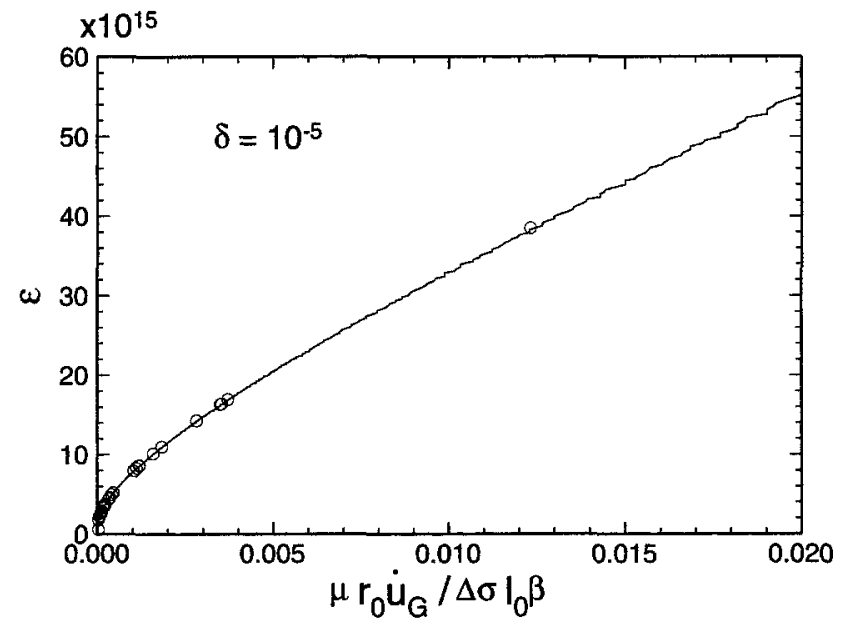

Figure 13. The constant of proportionality, $\varepsilon$, in equation (48) corrected for the level of ground noise. Circles indicate the data taken from Beroza and Ellsworth (1996).

dynamic stress drop and the nucleation time listed in Beroza and Ellsworth (1996) assuming that their definition of nucleation time is similar to ours. Figure 14 plots $l_{0}$ thus estimated against the nucleation moment estimated by Beroza and Ellsworth (1996). Because the noise levels are not available in the dataset, we assumed $\dot{u}_{G}=10^{-4} \mathrm{~cm} / \mathrm{sec}$ for all events. The estimated $l_{0}$ roughly scales with cube root of the observed nucleation moment. The estimates of $l_{0}$ obtained assuming $\dot{u}_{G}=10^{-3} \mathrm{~cm} / \mathrm{sec}$ are also shown for comparison. Then we determined $\varepsilon$ from the theoretical curve shown in Figure 13 and computed the nucleation moment $M_{0}^{v}$ using (48). Figure 15 compares $M_{0}^{v}$ thus calculated with those estimated by Beroza and Ellsworth (1996).

Figures 14 and 15 imply that if the definition of nucleation time adopted by Beroza and Ellsworth (1996) is similar to that in the present article, the observed dependence of the nucleation moment on the nucleation time can be interpreted as a consequence of the Spontaneous model with variable $l_{0}$. What we demonstrate here is that the Spontaneous model can produce a systematic trend between the nucleation time and the nucleation moment. The remaining question is whether our definition of nucleation time is comparable to that of Ellsworth and Beroza (1995) and Beroza and Ellsworth (1996). Because no objective criterion is used for the determination of nucleation time of observed seismograms, the answer to this question is not obvious. For some seismograms displayed in Figure 7 of Beroza and Ellsworth (1996) (e.g., numbers 11, 17, 21, and 23), the definition seems to be similar to ours. The synthetic seismograms for these events are shown in Figure 16. As a whole, they simulate the observed seismic nucleation phases very well. However, for some other events [e.g., numbers 1, 2, 3, 4, and 6, also the four larger events in Fig. 2 of Ellsworth and Beroza (1998)], the definition is different. For these events, the ground-motion velocity increased rapidly but later ex-

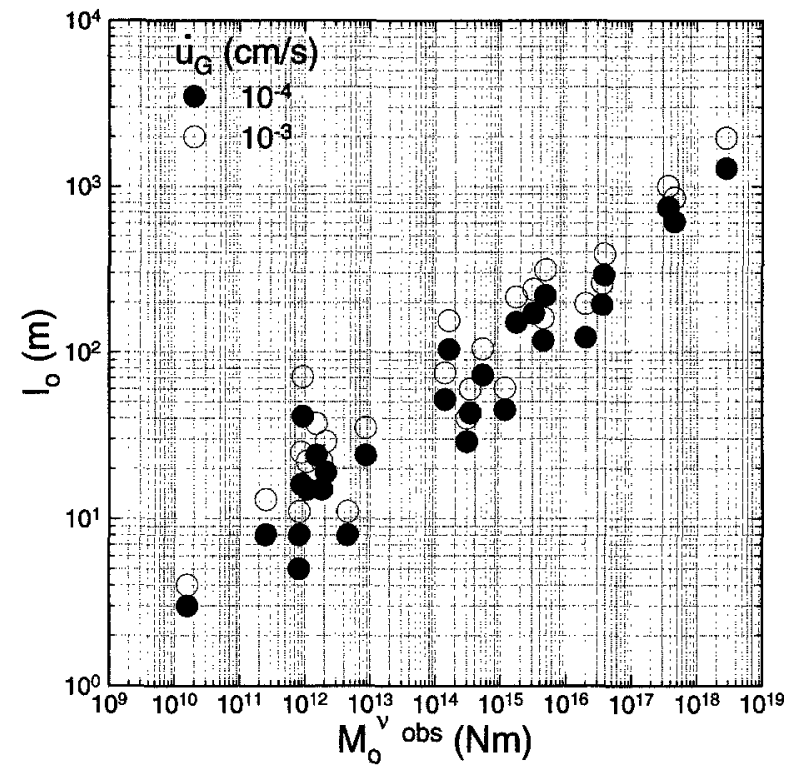

Figure 14. Estimated length of pre-existing crack plotted against observed seismic nucleation moment. Data are taken from Beroza and Ellsworth (1996). Solid and open circles denote the cases for the ground-noise level $\dot{u}_{G}=10^{-4}$ and $10^{-3} \mathrm{~cm} / \mathrm{sec}$, respectively.

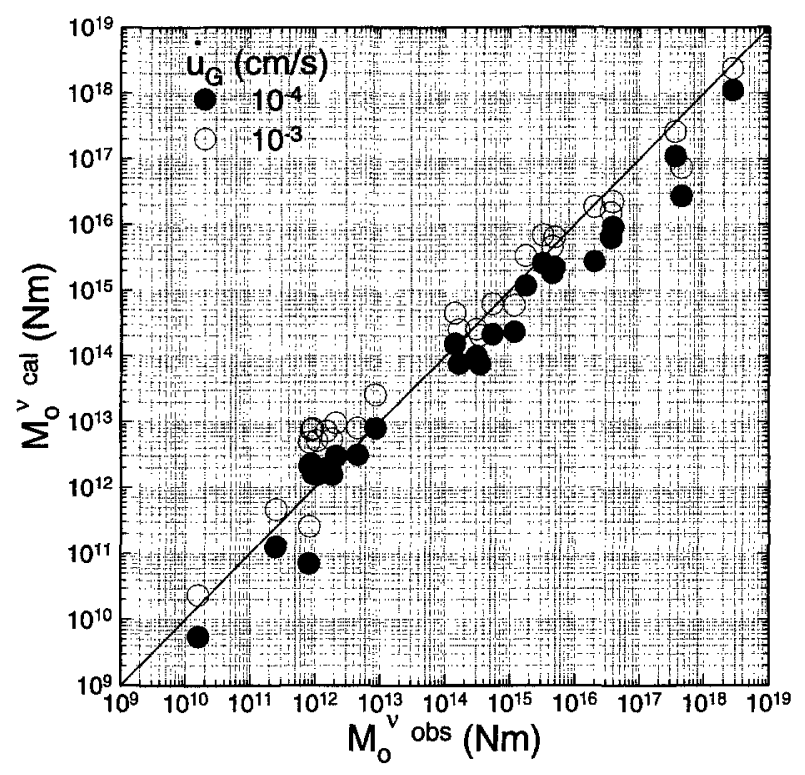

Figure 15. Comparison between calculated and observed nucleation moments. Data are taken from Beroza and Ellsworth (1996). Solid and open circles denote the cases for the ground-noise level $\dot{u}_{G}=$ $10^{-4}$ and $10^{-3} \mathrm{~cm} / \mathrm{sec}$, respectively.

hibited some hesitation (Ellsworth and Beroza, 1998), and the nucleation time is defined by the time of hesitation. This hesitation in waveform could be caused by multiple crack interactions or irregular rupture propagation due to heterogeneous fracture strength rather than the characteristics of 

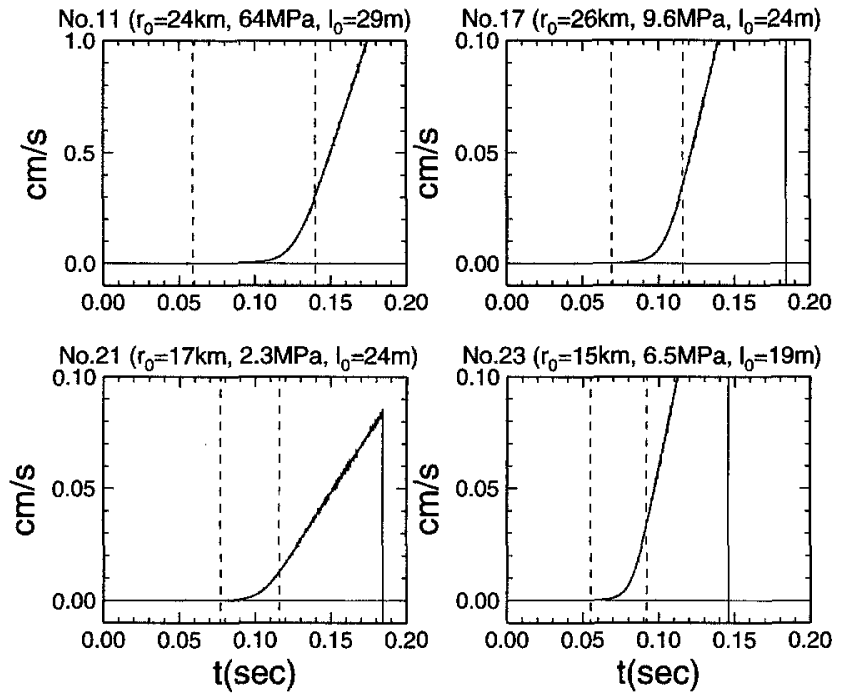

Figure 16. Synthetic velocity waveforms for some events listed in Beroza and Ellsworth (1996). Two dotted lines indicate the start and end of slow initial phase defined in the present model. It is assumed that $\dot{u}_{G}=10^{-4} \mathrm{~cm} / \mathrm{sec}$, and $\delta=10^{-5}$.

the smooth growth of a crack. Thus, it is unclear at present whether the relation between the earthquake magnitude and the duration of nucleation phase observed by Ellsworth and Beroza (1995), Beroza and Ellsworth (1996), and Ellsworth and Beroza (1998) is a manifestation of the Spontaneous model or not. To resolve this problem, it would be necessary to introduce a more objective criterion for the definition of the nucleation time of observed seismograms.

Several investigators have argued that the duration of slow initial phase (Iio, 1992) or the seismic nucleation time (Ellsworth and Beroza, 1995, Beroza and Ellsworth, 1996) scales with the eventual size of the earthquake. If this is the case, it would follow that the eventual size of the earthquake scales with $l_{0}$ of the Spontaneous model. Shibazaki and Matsu'ura (1998) proposed a similar scaling relation between the eventual size of earthquake and the critical size of nucleation zone in their model of earthquake nucleation using an empirical relation between the slow initial phase and the earthquake magnitude given by Umeda (1992). However, in view of the ambiguity of the definition of seismic nucleation phase, it is premature to conclude that the critical size of pre-existing crack, or the critical size of nucleation zone, scales with the eventual size of earthquake. In the Griffith model, rupture propagation is arrested at length $l$ if the rupture front encounters a barrier for which the surface energy exceeds

$$
\gamma_{B}=\frac{\left(\sigma_{0}-\sigma_{d}\right)^{2} \pi l_{0} U^{2}(\zeta)}{4 \mu}
$$

Because $U^{2}$ is very nearly a linear function of $\zeta$ (Rose, 1976),

$$
U^{2} \sim 1+\left(\frac{4}{5}\right) \zeta
$$

(49) can be rewritten as

$$
\begin{aligned}
\gamma_{B}=\frac{\left(\sigma_{0}-\sigma_{d}\right)^{2} \pi l_{0}\left(1+\frac{4}{5} \zeta\right)}{4 \mu} & \\
& =\frac{\left(\sigma_{0}-\sigma_{d}\right)^{2} \pi l\left(\frac{4}{5}+\frac{1}{5} l_{0}\right)}{4 \mu} .
\end{aligned}
$$

For $l \gg l_{0}, \gamma_{B}$ given in (51) is approximately proportional to $\left(\sigma_{0}-\sigma_{d}\right)^{2} l$ and does not depend on $l_{0}$. Thus, no simple model can explain the relation between $l$ and $l_{0}$, that is, the scale of the nucleation process and the eventual size of an earthquake.

\section{Discussion and Conclusions}

In our model, the rupture starts to grow from every edge of a pre-existing crack simultaneously. This may be an oversimplification of an actual earthquake initiation process. If both fracture strength and initial tectonic stress are heterogeneous in the real Earth, the initial rupture would occur at one point of pre-existing crack where the fracture condition is first satisfied. Due to high stress concentration near the tip of the pre-existing crack, the rupture may then propagate quickly along the rim of the pre-existing crack and thereafter grow outward more or less equidimensionally, as was demonstrated in some numerical experiments (Kostrov and Das, 1983, 1988; Fukuyama and Madariaga, 1997). If the rapid rupture along the rim of pre-existing crack is almost instantaneous relative to the time taken by a crack tip to advance by the same distance in the radial direction, the earthquake initiation process can approximately be described with the present model. If the transient process cannot be ignored and has to be incorporated explicitly in simulating the seismic initiation phase, we cannot handle the problem analytically and must have a recourse to numerical approach. At least, we can state that the bump prominent in the Trigger model (Fig. 8) would disappear if the rupture starts from a point and does not grow from the circular edge of pre-existing crack simultaneously.

In this article, the surface energy was assumed to be a material constant. Andrews (1976) showed that the effective surface energy increases with the crack length if anelastic deformation outside the main slip plane is included. If we introduce the surface energy increasing with the crack length into the Griffith's fracture criterion, the rupture velocity would not increase as fast as in the case of constant surface energy. It may happen that the rupture terminates before the rupture velocity attains its limiting value. The properties of 
seismic initiation phase would then become significantly different from those described in the preceding sections.

The preslip model proposed by Ellsworth and Beroza (1995) is similar to our model in a sense that it involves an aseismic nucleation zone that corresponds to the pre-existing crack in our model. Whereas the preslip model does not describe the waveform pattern of the seismic nucleation phase explicitly, our model predicts two types of seismic initiation phases, the slow initial phase of the type observed by lio (1995) on one hand and the abrupt onset of the type reported by Mori and Kanamori (1996) on the other.

We started with the assumption that the effects of structural complexity and multiple crack interactions are less important during the beginning of an earthquake and modeled its rupture process with a smooth growth of a single crack. This model generates a smooth waveform no matter which one of the two types of initial phases appears first. The seismic nucleation phases investigated by Ellsworth and Beroza (1995) and Beroza and Ellsworth (1996) are sometimes irregular, suggesting the complex rupture process from the start of an earthquake. If the degree of complexity is moderate, the seismic radiation can be simulated by superposing small ripples on the main phase due to a smooth growth of a single crack. If the degree of complexity is such that the effect of interaction of multiple cracks dominates over the smooth phase due to a smooth growth of a single crack, we must deal with the problem in a totally different framework. These complex processes are outside the scope of the present article. Coalescence of hierarchical sets of cracks (Fukao and Furumoto, 1985; Rice and Ben-Zion, 1996) may explain both the erratic nature of seismic nucleation phase and the scaling relation between source parameters for the seismic nucleation phase and source parameters for the entire earthquake (Ellsworth and Beroza, 1995; Beroza and Ellsworth, 1996). At least, we can state that each crack in the multiplecrack system would have its own mode of beginning depending on the trigger factor defined in the present article.

In conclusion, a rupture of a single crack based on the Griffith's fracture criterion can generate various types of seismic initiation phases depending on the trigger factor. We envisage that the trigger factor can vary in a wide range in the heterogeneous crust. Because the critical size of the preexisting crack, $l_{0}$, of the Spontaneous model scales with the nucleation moment, it should scale with the eventual size of the earthquake, if the proportionality of the nucleation moment and the eventual size holds, as suggested by Ellsworth and Beroza (1995). However, a theoretical consideration of rupture arrest by barriers using the Griffith's fracture criterion does not support this type of scaling relation.

\section{Acknowledgments}

We thank Y. Tio, B. Shibazaki, E. Fukuyama, J. Mori, T. Mikumo, S. K. Singh, R. Madariaga, and W. L. Ellsworth for valuable discussion. We are grateful to Yehuda Ben-Zion for a constructive review. This research was done while one of the authors (T.S.) visited the Division of Geological and
Planetary Sciences at the California Institute of Technology as a research fellow supported by the Japanese Ministry of Education. The DGPS Contribution Number is 8538 . This research was partially supported by the Southern California Earthquake Center. SCEC is funded by NSF Cooperative Agreement EAR-8920136 and USGS Cooperative Agreements 14-08-0001A0899 and 1434-HQ-97AG01718. The SCEC Contribution Number is 434.

\section{References}

Andrews, D. J. (1976). Rupture propagation with finite stress in antiplane strain, J. Geophys. Res. 81, 3575-3582.

Andrews, D. J. (1985). Dynamic plain-strain shear rupture with a slipweakening friction law calculated by a boundary integral method, Bull. Seism. Soc. Am. 75, 1-21.

Andrews, D. J. (1994). Dynamic growth of mixed-mode shear cracks, Bull. Seism. Soc. Am. 84, 1184-1198.

Beroza, G. C. and W. L. Ellsworth (1996). Properties of the seismic nucleation phase, Tectonophysics 261, 209-227.

Das, S. (1981). Three-dimensional spontaneous rupture propagation and implications for the earthquake source mechanism, Geophys. J. 67, 375-394.

Day, S. M. (1982). Three-dimensional simulation of spontaneous rupture: The effect of nonuniform prestress, Bull. Seism. Soc. Am. 72, 18811902.

Dieterich, J. H. (1978). Preseismic fault slip and earthquake prediction, $J$. Geophys. Res. 83, 3940-3948.

Ellsworth, W. L. and G. C. Beroza (1995). Seismic evidence for an earthquake nucleation phase, Science 268, 851-855.

Ellsworth, W. L. and G. C. Beroza (1998). Observation of the seismic nucleation phase in the Ridgecrest, California, earthquake sequence, Geophys. Res. Lett. 25, 401-404.

Eshelby, J. D. (1957). The determination of the elastic field of an ellipsoidal inclusion and related problems, Proc. R. Soc. London A241, 376396.

Fukao, Y. and M. Furumoto (1985). Hierarchy in earthquake size distribution, Phys. Earth Planet. Interiors, 37, 149-168.

Fukao, Y. and B. Shibazaki (1995). Is the eventual size of earthquake determined by the mode of earthquake initiation?, Kagaku 65, 211-218.

Fukuyama, E. and R. Madariaga (1997). Break of an asperity, Abstracts of the SSJ Meeting, No. 2, A12.

Haskell, N. (1964). Total energy and energy spectral density of elastic wave radiation from propagating faults, Bull. Seism. Soc. Am. 56, 18111841.

Ida, Y. (1972). Cohesive force across the tip of a longitudinal-shear crack and Griffith's specific surface energy, J. Geophys. Res. 77, 37963805.

Iio, Y. (1992). Slow initial phase of the P-wave velocity pulse generated by microearthquakes, Geophys. Res. Lett. 19, 477-480.

Iio, Y. (1995). Observations of the slow initial phase generated by microearthquakes: implications for earthquake nucleation and propagation, J. Geophys. Res. 100, 15333-15349.

Ishihara, Y., Y. Fukao, I. Yamada, and H. Aoki (1992). Rising slope of moment rate functions: the 1989 earthquakes off east coast of Honshu, Geophys. Res. Lett. 19, 873-876.

Kame, N. and T. Yamashita (1997). Dynamic nucleation process of shallow earthquake faulting in a fault zone, Geophys. J. Int. 128, 204-216.

Knopoff, L. (1958). Energy release in earthquakes, Geophys. J. R. Astr. Soc. 1, 44-52.

Kostrov, B. V. (1964). Self-similar problems of propagation of shear cracks, PMM 28, 889-898.

Kostrov, B. V. (1966). Unsteady propagation of longitudinal shear cracks, J. Appl. Math. Mech. 30, 1241-1248.

Kostrov, B. V. and S. Das (1983). Breaking of a single asperity: rupture process and seismic radiation, J. Geophys. Res. 88, 4277-4288.

Kostrov, B. V. and S. Das (1988). Principles of Earthquake Source Mechanics, Cambridge U. Press, Cambridge, 286 pp. 
Miyatake, T. (1980). Numerical simulations of earthquake source process by a three-dimensional crack model. Part I. Rupture process, J. Phys. Earth 28, 565-598.

Mori, J. and H. Kanamori (1996). Rupture initiations of microearthquakes in the 1995 Ridgecrest, California sequence, Geophys. Res. Lett. 23, 2437-2440.

Nakatani, M., B. Shibazaki, S. Kaneshima, Y. Fukao, M. Matsu'ura, N. Kame, Y. Sugioka, R. Ootani, T. Ito, and M. Takeo (1995). A correlation of the earthquake magnitude with the properties of seismic initiation phases observed by a high-gain recording system, Monthly Ckуии 17, 125-129.

Ohnaka, M. and Y. Kuwahara (1990). Characteristic features of local breakdown near a crack-tip in the transition zone from nucleation to unstable rupture during stick-slip shear failure, Tectonophysics 175, 197220.

Palmer, A. C. and J. R. Rice (1973). The growth of slip surfaces in the progressive failure of over-consolidated clay, Proc. R. Soc. London, A 332, 527-548.

Rice, J. R. and Y. Ben-Zion (1996). Slip complexity in earthquake fault models, Proc. Natl. Acad. Sci. USA 93, 3811-3818.

Rose, L. R. F. (1976). On the initial motion of a Griffith crack, Int. J. Fract. 12, 829-841.

Sato, T. (1994). Seismic radiation from circular cracks growing at variable rupture velocity, Bull. Seism. Soc. Am. 84, 1199-1215.

Sato, T. and T. Hirasawa (1973). Body wave spectra from propagating shear cracks, J. Phys. Earth 21, 415-431.

Shibazaki, B. and M. Matsu'ura (1998). Transition process from nucleation to high-speed rupture propagation: scaling from stick-slip experiments to natural earthquakes, Geophys. J. Int. 132, 14-30.

Shibazaki, B., Y. Yoshida, T. Ito, T. Hatori, N. Kobayashi, S. Kaneshima, and Y. Fukao (1994). Initial rupture process of the aftershocks of the 1993 Hokkaido-Nansei-oki earthquake, Abstracts of SSJ Meeting, No. 2, 276.

Umeda, Y. (1990). High-amplitude seismic waves radiated from the bright spot of an earthquake, Tectonophysics 141, 335-343.

Umeda, Y. (1992). The bright spot of an earthquake, Tectonophysics 211, 13-22.

Umeda, Y., T. Yamashita, T. Tada, and N. Kame (1996). Possible mechanisms of dynamic nucleation and arresting of shallow earthquake faulting, Tectonophysics 261, 179-192.

Virieux, J. and R. Madariaga (1982). Dynamic faulting studied by a finite difference method, Bull. Seism. Soc. Am. 72, 345-369.

Department of Earth and Environmental Sciences

Hirosaki University

3 Bunkyo-cho

Hirosaki, Japan

(T. S.)

Seismological Laboratory 252-21

California Institute of Technology

Pasadena, California 91125

(H. K.)

Manuscript received 20 April 1998. 